\title{
Withdrawal of neonatal intensive care in the home
}

\author{
J M Hawdon, S Williams, A $M$ Weindling
}

Despite rapid and significant advances in neonatal care, many conditions still prove lethal. Under these circumstances, it is acceptable to withdraw intensive support when it is clear that there is no chance of recovery. ${ }^{1}$ In other fields, such as paediatric oncology, much effort is devoted to allowing terminally ill children to spend their last few hours or days at home with those who love them. The possibility of allowing newborn babies, who have been receiving intensive care, home to die has not been widely explored.

We present the histories of three patients cared for within a six month period to illustrate that in certain circumstances withdrawal of intensive care may take place in the home, so that the death can occur in privacy and in circumstances that the family find more comforting.

\section{Case reports}

CASE 1

A baby girl was conceived after a prolonged period of infertility. She was delivered at 28 weeks' gestation by caesarean section because of intrauterine growth retardation and abnormal umbilical artery blood flow detected by Doppler ultrasonography. She was extremely small: her birth weight was $570 \mathrm{~g}(<3 \mathrm{rd}$ centile), her head circumference was also less then the 3rd centile, and she was noted to be dysmorphic. No specific syndrome was identified but she was found to have an abnormal structural rearrangement of one $\mathrm{X}$ chromosome. She was ventilated from birth, initially for respiratory distress syndrome but later her respiratory insufficiency was thought to be secondary to a combination of eventration of the right hemidiaphragm and central or neuromuscular respiratory failure. Ten trials of extubation failed. She was fully enterally fed from day 45 postnatally.

At the age of 19 weeks (that is, seven weeks after term) it was apparent that the baby would have no chance of existence independent of the ventilator and withdrawal of intensive support was discussed. Her parents requested that this should occur at home. The baby was transferred home ventilated in the 'flying squad' transport incubator, accompanied by an experienced paediatric registrar and nursing sister. Portable suction apparatus was provided by the hospital and her general practitioner (GP) prescribed a portable oxygen cylinder which was delivered to her home by a local pharmacist. The baby was extubated on arrival at home and nursed with facemask oxygen. The nursing sister remained with the parents until after the baby's death eight hours later and the consultant paediatrician visited shortly afterwards to certify the death. Funeral directors returned the baby to hospital pending funeral arrangements.

\section{CASE 2}

A baby girl was delivered at 30 weeks' gestation by caesarean section after placental abruption and cardiotocographic evidence of fetal distress. Her birth weight was $1500 \mathrm{~g}$ (50th centile) and her head circumference was on the 90th centile. She was ventilated for respiratory distress syndrome for nine days and her postnatal course was complicated by bilateral intraventricular haemorrhage with parenchymal extension and subsequent hydrocephalus requiring repeated ventricular taps. She was never fully enterally fed. At the age of 7 weeks her general condition deteriorated, oxygen requirements increased, and she developed hepatosplenomegaly and signs of portal hypertension. This was later demonstrated at necropsy to be secondary to infective thromboembolism in the inferior vena cava.

As the baby's condition worsened and the poor prognosis became apparent, and after discussion with her parents, it was felt inappropriate to institute ventilatory support and her parents decided to take her home. Her GP prescribed portable oxygen and the family was supported by regular visits from the hospital community liaison sister and the GP. The baby died two days later and the death was certified by the GP. The baby was returned to hospital by the funeral directors for postmortem examination.

CASE 3

A baby girl was born at 31 weeks' gestation by spontaneous vertex delivery. Her parents were first cousins and her father had haemophilia. The baby's birth weight was $1620 \mathrm{~g}$ (50th centile) and her head circumference was greater than the 50th centile. She was noted to be dysmorphic and had flexion contractures of the limbs. She did not at first require ventilatory support but became progressively less active and was ventilated (in air) from the fifth postnatal day because of respiratory 
insufficiency of central or neuromuscular origin. She received no enteral feeds.

At the age of 14 days the baby was still ventilator dependent with no spontaneous movements. After discussion with her parents the decision was made to transfer her home, ventilated in the flying squad incubator, accompanied by an experienced staff nurse (chosen by her parents). She was extubated at home and died one hour later. The paediatric senior registrar visited to certify death and bring the baby back to hospital (carried in the nurse's arms) for postmortem examination.

\section{Discussion}

We felt that when the outlook for a sick baby was hopeless, the parents and perhaps their baby benefited from being allowed to go home to die. For example, the parents appreciated the opportunity to 'show the baby' the nursery they had prepared, to bath and dress her and to take photographs or video recordings, in the same way as any parent taking a new baby home. None of these babies had siblings, but for other families there may be the added advantage of allowing siblings to see the baby away from potentially frightening hospital surroundings and better understand her death. Privacy and comfort are facilities that should be made available to parents when children die in hospital, but the parents described above felt these needs were best met by leaving the hospital. Under these circumstances, it is important that the families should not be deprived of the same support by familiar and experienced staff that they would have received in hospital. This support should be offered without intrusion of privacy. We feel that this was amply provided in each case as each family was accompanied by a member of staff with whom they had developed a rapport during their stay in hospital.

There are other prerequisites for allowing babies home to die. The baby must be stable enough for transfer home. This should be at the parents' request or on their acceptance of an offer gently made; the situation should not be forced upon them. Home oxygen and suction and possibly the provision for gavage feeds should be provided if death is not thought to be imminent. There must be provision for a medical practitioner who has seen the baby alive to certify the death and arrangements should be made for transfer of the baby after death. It may be helpful to discuss the possibility of necropsy before the baby dies and the Department of Health leaflet Guide to the Postmortem Examination may aid this discussion. ${ }^{2}$ Finally, continuing support and counselling and the explanation of postmortem examination results should be available to all parents, and staff involved should receive support and feedback. In the cases discussed, the events surrounding each baby's death were viewed positively by staff and families.

These case histories illustrate the need for a multidisciplinary approach for the withdrawal of intensive care at home. The role of GPs in such circumstances needs further exploration. They may prescribe portable oxygen or other necessary drugs and may offer valuable support. However, in order to certify death they must have seen the baby alive. Therefore, planning and close liaison between hospital and community are important.

We hope that this service may be provided more often in such uncommon but tragic circumstances.

1 Doyal L, Wilsher D. Towards guidelines for withholding and withdrawal of life prolonging treatment in neonatal medicine. Arch Dis Child 1994; 70: F66-70.

2 Department of Health. Guide to postmortem examination - brief notes for parents and families who have lost a baby in pregnancy or early infancy. London: Department of Health, 1993.

\section{Commentary}

This article by Hawdon et al is welcome in so far as it focuses on the important issue of the management of the infant for whom intensive care has ceased to offer hope of a satisfactory outcome. Thankfully, neonatology is now a mature enough specialty for the withdrawal of intensive care in these circumstances to be an acceptable course of action. However, we do not always manage this situation with the same meticulous attention to detail that is given to the maintenance of life support.

The British Paediatric Association classification of intensive care recognises the needs of the dying infant, and family, by categorising the last day of life as requiring level 1 intensive care. To an extent this reflects the need to have a dedicated nurse available but it also emphasises the demands which the elective withdrawal of intensive care makes on the whole neonatal unit team and on the resources which it commands. Whether or not this care and support for baby, parents, and staff, can be provided equally well in the home environment must be highly debatable. Great caution should be exercised in extrapolating from what might have been a successful venture in the cases reported to the general case.

It is also difficult to see how it can be in the baby's best interests to be transported for the purpose of dying elsewhere. The babies in question are very sick and the extra handling and ambulance journey cannot be pleasant for them.

Finally, there are resource implications. Most units find it a strain to provide a transport team and taking doctors and nurses out of the unit always has an opportunity cost attached to it, which must be borne by other infants.

It may be that there are circumstances in which the trade-off between these disadvantages and perceived benefits seems sufficiently favourable to justify the practice reported by Hawdon et al. In general, though, our energies would be better employed in trying to improve all aspects of our management of the dying baby in hospital.

Creating appropriate facilities for the family of the dying baby is clearly most important. A room adjacent to the neonatal unit and furnished in a domestic style is ideal. Most 
units should be able to provide this, and many probably have, with relatively minor resource implications. In this setting, the involvement of senior medical staff to reassure and support parents or junior colleagues is easily invoked and is of considerable value. Attention to these factors will provide an environment within which the immediate and extended family can spend time with the dying infant in privacy, but with the close support of the medical and nursing team.

P R F DEAR

St fames's University Hospital, Leeds LS9 $7 T F$ 